\title{
XXVII.
}

\section{Grosser sarcomatöser Tumor in der Pituitargegend.}

\author{
Mitgetheilt von Carl Ernst Emil Hoffmann, \\ Prosector und Docent in Giessen.
}

(Hierzu Taf. VI.)

\begin{abstract}
Da Geschwülste in der Pituitargegend zu den verhältnissmässig seltener vorkommenden pathologischen Neubildungen gehören, so halte ich einen von mir genauer untersuchten Fall *) nicht ohne Interesse.
\end{abstract}

Elisabetha R. wurde am 13. Januar 1861 in die hiesige Gebäranstalt aufgenommen.

Sie war im Januar 1832 als Kind sebr armer Eltern im Vogelsherge geboren, zeichnete sich wăhrend ihres Schulbesuches durch Fleiss und Fassungskraft aus, so dass sie stets einen der obersten Plätze unter ihren Mitschülerinnen einnahm. Nach ihrem Austritte aus der Schule war sie als Magd fast ohne Unterbrechung in Diensten; auch in dieser Zeit zeigte sie sich stets als ein heiteres, aufgewecktes, gesundes Mädchen, an welcher Geistes- oder Sinnesstörungen niemals beobachtet wurden.

Vor mehreren Jahren war sie an Typhus erkrankt, nach Ablauf desselben jedoch wieder völlig gesund. Sie hatte zweimal leicht geboren und war auch Schwangerschaft und Wochenbett in beiden Fällen ganz normal verlaufen. Im Verlaufe der letzten Schwangerscbaft klagte sie öfters ïber heftigen Kopfschmerz, der sich bis tief in die Orbitalgegend hin erstreckte, mit bedeutendem Blutandrange nach dem Kopfe verbunden war und oft so heftig wurde, dass sich die Kranke Tage lang zu Belte legen musste, dabei war sie auffallend in sich gekehrt und einsilbig. Gegen das Ende der Schwangerschaft trat ausser diesem heftigen Kopf-

*) Ueber diesen Fall findet sich bereits eine Mittheilung unter der Aufschrift: "Fall von Eclampsia puerperalis in Verbindung mit einem Fungus durae matris" von Dr. F. A. Kehrer (Sohn des früheren Assistenten an hiesiger Gebäranstalt), in der Monatsschrift für Geburtskunde. 1861. Bd. XVIII. Die Section wurde von dem seitherigen zweiten Assistenten des anatomisch-physioIogischen Instituts Dr. Fehr angestellt, von welchem ich gleich nach der Section die mitgetheilten Sectionsresultate erhielt, während mir die Präparate gleichfalls unmittelbar nachber zu Gebote standen. Die Anamnese verdanke ich der gütigen Mittheilung des Herrn Pfarrer Schaub zu Ulrichstein, die Krankengeschichte der Güte des Herrn Geheimraths von Ritgen. 
scbmerze öfters Schwindel, Flimmern vor den Augen und Gesichtsschwäche auf, welch' letztere mit Strabismus internus der beiden Seiten (etwas stärker rechts) verbunden war.

Die Geburt verlief aucl diesmal ziemlich regelmässig. Nach dreistiundigen leichten Wehen gebar sie am 23. Januar Morgens 2 Uhr einen gesunden Knahen; die Nachgeburt falgte leicht, der Blutverlust war unbedeutend. Die beiden ersten Tage des Wochenbettes verliefen gut, nur war die Kranke sehr theilnahmlos und starrte ruhig vor sich bin. Strabismus unverändert, Kopfschmerz und Schwindel fehlten.

Am 24. Januar Abends $9 \frac{1}{2} \mathrm{Uhr}$ heftiger Schüttelfrost mit nachfolgender Hitze und frequentem kleinen Pulse. Starker Meteorismus und Schmerzhaftigkeit des Leibes.

Am 25. Januar Morgens. Puls 120. Kopf ohne hervorragende Schmerzen; Leib aufgetrieben, schmerzhaft. Abends $10 \frac{1}{2}$ Uhr zweiter Schüttelfrost mit darauf folgendem allgemeinen clonischen Krampfanfalle, der vorzugsweise stark die rechten Extremitäten, den Kopf und Hals ergriff, wăhrend Rumpf und linke Extremitäten weniger davon befallen waren. Der Kopf wurde ruckweise Anfangs nach der linken, später nach der rechten Seite, gewölnlich während der Inspiration gedreht; dazu gesellten sich mimische Gesichtskrämpfe und Schlingkrämpfe, der Speichel floss aus dem Munde, die Zunge wurde nicht vorgestreckt. Das Gesicht war geröthet, die Augenlider zuckten und die Augäpfel wurden krampfhaft bewegt, wäbrend die engen Pupillen gegen Lichtreiz nicht reagirten. Puls 140 Schläge, Haut anfangs trocken heiss, dann mehr klebrig.

Dieser Krampfanfall wiederholte sich bis um $12 \mathrm{Jhr}$ Nachts 10mal, dann Ruhe bis um 1 Uhr, dann bis 3 Uhr Morgens 6 weitere Anfälle, welche jedoch immer schwächer wurden, bis um 3 Vhr der Tod erfolgte. In den krampffreien Zeiten Sopor. Angenlider halb offen. Pupillen contrahirt. Die letzteren erweiterten sich rasch nach dem Aufhören des Herzschlages.

Section nach 30 Stunden.

Ziemlich kräftiger schlanker Körperbau. Ueber den ganzen Körper, namentlich auf dem Rücken, starke Todtenflecke, Bauchdecken grün, Leichenstarre vorzugsweise an den unteren Extremitäten stark entwickelt.

Die Schädeldecke trennt sich leicht von der Dura mater, ist von mittlerer Dicke, an der Innenfläche ziemlich glatt und zeigt nur zu beiden Seiten der Sutura sagittalis, sowie an dem Stirnbeine einige starke Vertiefungen bedingt durch grosse Pacchionische Granulationen. In der Umgebung dieser Stellen ist die Dura mater stark verdickt, sonst ist sie prall gespanint und die Blutgefässe mässig gefüllt. In dem Sinus longitudinalís wenig dünnllüssiges dunkles Blut mit spärlichen Speckhautgerinnseln. Die weichen Häute sind mit Ausnahme der Pacchionischen Wucherungen ohne wesentliche Veränderungen, die Gefässe der Gehirnoberfläche ziemlich gefüllt. Die Gyri von mittlerer Entwicklung.

Bei der Herausnahme des Gehirns findet sich die Basis in der Gegend des Chiasma nervorum opticorum sehr stark durch eine in dieser Gegend auf der Schädelbasis aufliegende Geschwulst eingedrückt, die vorderen Hirnlappen aach oben und den Seiten verdrängt. Die Tractus optici waren mit dem Chiasma durch die 
Geschwulst nach oben und seitwärts verdrängt and hatten einen bogenförmigen Verlauf. Die Anfänge der Sehnerven waren platt, die Hypophysis cerehri war durch die Geschwulst aus der Sella turcica heransgedrängt und lag oben auf ihr. Die Nervi oculomotorii sind verlängert und dünner als gewöhnlich, wenigstens soweit sie durch die Geschwulst aus der Lage gedrängt sind.- Die Nervi olfactorii waren comprimirt, namentlich war der linke Riechkolben stark geschrumpft. Die Corpora mamillaria platt, ebenso die Gyri in der Ungebung der Fossa Sylvii, namentlicb rechterseits. Die Gehirnsubstanz ist mässig hyperämisch, die Seitenventrikel nicht wesentlich in Form und Grösse verändert, nur das Unterhorn der rechten Seite erscheint etwas zusammengedrückt; sie enthalten eine geringe Quantïtät rötblich seröser Fliissigkeit. Von der Schädelbasis aus der Mitte der Sella turcica erhebt sich ein runder, blassröthlicher Tunor von höckerig-hïgeligem, etwas gelapptem Anseben und etwas derberer Consistenz als die Gehirnmasse (s. Fig. 1). Er entspringt mit schmaler Basis von der Dura mater in der Tiefe der Sella turcica, mit welcher er fest verwachsen ist. In seinem grössten Querdurchmesser $48 \mathrm{Mm}$., im grössten Längsdurchmesser $42 \mathrm{Mm}$. und erbebt sicb über die angrenzenden Theile der Schädelbasis um $34 \mathrm{Mm}$.

Er liegt nicht symmetrisch auf der Sella turcica auf, sondern ragt $2 u \frac{3}{4}$ auf die rechte, zu $\frac{1}{4}$ auf die linke Seite ïber die Mittellinie, während er nach vorn $6 \mathrm{Mm}$. von dem hinteren Ende der Crista galli entfernt liegt und nach hinten etwa ebenso weit das Dorsum sellae turcicae überragt, auf dessen obere Abtheilung er sich mit seiner hinteren Partie auflegt. Das Neurilem des Sehnerven, sowie die Dura mater der die Geschwulst begrenzenden Knochentheile waren durch feine Faserzüge an die mit den weichen Hirnhäıten andererseits zusammenbängende und scheinbar zu dieser gehörende feine Membran angeheftet, welche den Tumor überzog. Bei genauerer Untersuchung erscheint die Masse nur in der Tiefe der Sella tureica mit schmaler Basis mit der Dura mater verwachsen, wäbrend sich nirgends ein Zusammenhang mit den Knochen zeigt. Die Keilbeinsinus sind nicht wesentlich verändert, aber die Knochenabtheilungen an der vorderen wie hinteren Begrenzung der Sella torcica sind etwas geschwunden.

Der Rückenmarkskanal zeigt sowohl an der vorderen wie hinteren Seite starke Hyperämie, namentlich gegen das Ende hin, in dem Arachnoidealraume wenig seröse Flüssigkeit. Das Rückenmark selbst obne besondere Veränderung, graue und weisse Substanz nicht sehr scharf unterscheidhar.

Beide Lungen, namentlich in den unteren Partien, stark ödematös, in beiden Herzhälften neben einigem flüssigen dunklen Blute starke Fjbringerinnsel, die Pulmonalklappen gefenstert.

Die Gedärme meteoristisch aufgetrieben, die Leber mit drei Schnürstreifen, senst ebenso wie Nieren und Milz ohne wesentliche Abnormität. Erscheinungen uberstandener Peritonitis nicht vorhanden. Der Uterus blass, derb, misst im Querdurchmesser zwischen der Insertion der Tuben $137 \mathrm{Mm}$., im Längendurchmesser van dem Fundus bis zur Insertion der Scheide $198 \mathrm{Mm}$. Die Dicke der Uteruswand beträgt am Fundus $27 \mathrm{Mm}$, in der Mitte des Körpers $30 \mathrm{Mm}$., am Cervix $15 \mathrm{Mm}$. Die Oberfläche der Uterushöhle ist mit schleimigen, fetzigen, leicht blutig 
gefärbten Massen bedeckt, nirgends missfarbig, nirgends Spuren von Eiterung. Die Placentarinsertion vora, mitten und oben dunkelroth. Im rechten Ovarium ein Corpus luteum.

Ehle ich zu eituer Betrachtung über den Zusammenhang der Erscheinungen während des Lebens mit dem nach dem Tode constatirten anatomischen Befunde übergehe, liegt es mir noch ob, etwas über die feinere Struktur der vorliegenden Neubildung zu sagen.

Im Allgemeinen stimmt dieselbe ganz mit den Charakteren der fibroplastischen Geschwülste, wie sie Lebert in seiner bekannten Arbeit über die Gesehwälste im Hirn und seinen Hüllen *) angiebt. Ihrer Consistenz und Beschaffenheit nach würde sie mit Billroth*) zu den medullaren Faser-oder Spindelzellensarkomen und zwar zu der Form unter a, bei welchen das Gewebe aus langgestreckten Faserzellen ohne weitere Fortsătze besteht, zu rechnen sein. Der Tumor besteht nämlich nur aus sehr grossen und breiten, dabei äusserst zarten, langgestreckten Faserzellen, welche mit einer äusserst spärlichen Menge von Bindegewebsfasern ohne alle regelmässige Anordnung in ein dichtes Faserwerk mit einander verfilzt sind (Fig. 2 a.). Die Faserzellen selbst besitzen grosse deutliche Kerne mit körnigem Inhalte und einen oder auch zwei Kernkörperchen; sie lassen sich leicht und klar unter dem Mikroskope auch ohne Anwendung von Reagentien beobachten.

Die Beschaffenheit dieses Tumors scheint, ganz mit der des neulich von Grohe***) in Verbindung mit einer Cyste beschriebenen Tumors ïbereinzustimmen, nur war von einer rückgängigen Metamorphose nichts zu bemerken. Auch mir fielen an inm die ausserordentlich grossen und breiten, dabei äusserst feingebauten Zellen mit langen Ausläufern auf, wie sie in Fig. 2 a., b. und c. abgebildet sind.

Die Entstehung und Entwicklung dieser Geschwulst fällt aller Wahrscheinlichkeit nach in die Zeit der letzten Schwangerschaft, wie weit zuriick der Anfang derselben zu datiren ist, dürfte schwer

*) Siehe dieses Archiv Bd. III. S. 481.

**) Siehe dieses Archiv Bd. XVIII. S. 84.

***) Siehe dieses Archiv Bd. XXII. S. 451. 
zu ermitteln sein, da die während der Schwangerschaftsperiode aufgetretenen Kopfschmerzen gewiss nicht durch die ersten Anfänge der Geschwulst bedingt wurden, sondern einem schon vorgesehritteneren Stadium derselben entsprachen.

Betrachten wir die Erscheinungen vor den zwei letzten Tagen, so stimmen sie im Wesentlichen mit dem von Mohr mitgetheilten Falle über eine gänseeigrosse Geschwulst in der Pituitargegend*) uberein. Auch dort fanden sich periodischer Kopfschmerz, Flimmern vor den Augen, häufige Congestiverscheinungen zum Kopfe, Abnahme des Sehvermögens; dann mit Fall 1, 3 und 5 von Lebert $\left.{ }^{* *}\right)$.

Sebr ausgesprochen waren in unserem Falle der heftige Kopfschmerz bis tief in die Supraorbitalgegend hinein, die Congestionen nach dem Kopfe, das Schielen und endlich die Amblyopie. So dass sich auch in diesem Falle die vorzugsweisen Symptome wiederfinden, wie sie Friedreich in seiner Arbeit über die Geschwülste der Schädelhöhle ${ }^{* * *}$ ) als charakteristisch für die Geschwülste der Pituitargegend annimmt. Ein Symptom, das man nach dem anatomischen Befunde als vorhanden gewesen vermuthen sollte und das sich bei Fall 1 von Lebert $\dagger$ ) verzeichnet findet, nämlich Störung des Riechvermögens, wurde trotz der bedeutenden Verkümmerung der Olfactorii nicht beobachtet, doch liegt kein Grund vor, anzunehmen, dass diese Störung gefehlt habe, da einerseits die Aufmerksamkeit früher der Art der Erkrankung fast. nicht zugewendet worden war, andererseits aber Leute aus diesen Ständen selten viel Gewicht auf diesen Sinn legen, daher auch Veränderungen in der Funktion desselben nicht beachten oder selbst nicht beobachten.

Bei weitem am schwierigsten erscheint es, die Beziehungen

*) Mitgetheilt von Friedreich „in Geschwülste der Schädelhöhle“. Würzburg, 1853. S. 84 .

"*) Arehiv Bd. III. S. 532 u. 533.

***) Beiträge zur Lehre der Geschwülste innerbalb der Schädelböhle. Würzburg, 1853. S. 86.

†) 1. c. S. 532 . 
der Geschwulst zu den Erscheinungen, wie sie an den letzten Tagen vor dem Tode auftraten, richtig zu würdigen.

Da nicht wohl anzunehmen ist, dass die Wucherung der Neubildung ganz plötzlich während des Wochenbettes sehr bedeutend zugenommen habe und dadurch ein stärkerer Druck und Reiz als fruher auf das Gehirn bewirkt habe, so liegt es sehr nahe, anzunehmen, dass andere Momente als die nächste Ursache der so heftig auftretenden Convulsionen anzusehen seien. Da liegt es denn nun natürlich nahe, sie in Beziehung zu setzen zu der puerperalen Erkrankung des Uterus, die sich schon 24 Stunden vor Auftreten der Krämpfe zeigte, und diess un so mehr, als früher bei der Kranken niemals Neigung zu Krämpfen beobachtet worden war. Bedenkt man aber, dass verhältnissmässig selten diese Kranpferscheinungen in der Wochenbettsperiode auftreten ${ }^{*}$ ), so fällt zum mindesten die Heftigkeit der Erscheinungen auf, namentlich da die Sektion keine weiter bedeutenden causalen Momente, wie Nierenaffektionen u. s. w. ergeben hat, und auch die Lokalaffektion des Uterus keine bedeutenden anatomischen Veränderungen nach dem Tode mehr nachweisen liess. lch bin nach reiflicher Abwägung der Erscheinungen und des Befundes nicht abgeneigt, mich wenigstens in so weit der Kehrer'schen Ansicht**) anzuschliessen, als ich glaube, dass während sich der Tumor in früheren Perioden verhältnissmässig latent verhielt, er als die wirkende Ursache der bedeutenden Gehirnerscheinungen anzusehen ist, nachdem zuyor durch die puerperale Erkrankung eine gesteigerte Erregung dieses Centraloryanes bedingt worden war.

*) Siehe Hasse, Krankheiten des Nervenapparates in Virchow's Handbuch der Pathologie und Therapie. Bd.IV. 1. S. 291.

*) l. c. S. 234 . 

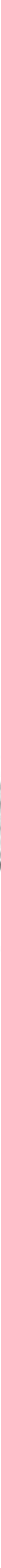

of Sebinge liter 